\title{
A Literature Review of Intrathecal Morphine Analgesia in Patients Undergoing Major Open Hepato-Pancreatic-Biliary (HPB) Surgery
}

\author{
Jefferson Zhi Jie Tang (iD ${ }^{1,{ }^{*}}$ and Laurence Weinberg (iD) ${ }^{1}$ \\ ${ }^{1}$ Department of Anaesthesia, Austin Hospital, Melbourne, Australia \\ "Corresponding author: Department of Anaesthesia, Austin Hospital, Melbourne, Australia. Email: jeffersonzjtang@gmail.com
}

Received 2019 May 25; Revised 2019 October 25; Accepted 2019 November 12.

\begin{abstract}
Context: The optimal analgesic method for patients undergoing major open hepato-pancreatic-biliary surgery remains controversial. Continuous epidural infusion at the thoracic level remains the standard choice, however concerns have been raised due to associated complications. Single shot intrathecal morphine has emerged as a promising alternative offering similar analgesia with an enhanced safety profile.

Evidence Acquisition: This review aimed to evaluate the literature comparing intrathecal morphine analgesia to other analgesic modalities following major open hepato-pancreatic-biliary surgery. The primary outcome was pain scores at rest and on movement $24 \mathrm{~h}$ after surgery. Secondary outcomes were postoperative opioid consumption within 72 postoperative hours, length of stay (LOS), intra-operative fluid administration and post-operative fluid administration within 72 postoperative hours, and overall systemic complication rate within 30 postoperative days.

Results: Eleven trials matching the inclusion criteria were analysed. Intrathecal morphine resulted in equivalent or lower pain scores when contrasted to alternative techniques, but required higher amounts of postoperative opioid. Intrathecal morphine also offered reduced LOS and reduced fluid administration requirements to epidural analgesia, and there was no difference observed in major complication rate between analgesic modalities.

Conclusions: In summary the evidence suggests that intrathecal morphine may be a better first-line analgesic modality than epidural analgesia in the context of major open hepato-pancreatic-biliary surgery, but high-quality evidence supporting this is limited.
\end{abstract}

Keywords: Liver, Surgery, Analgesia, Epidural, Intrathecal

\section{Context}

Major open hepato-pancreatic-biliary (HPB) surgeries have become performed with increasing frequency due to a rising incidence of major HPB diseases, notably hepatocellular carcinoma and pancreatic cancer $(1,2)$. Overall mortality of major HPB surgeries stands at less than six percent, however complication rates (and thus morbidity) has remained high at over $25 \%$ (3). Accordingly, enhanced recovery after surgery (ERAS) programs have become widespread in the last decade due to their demonstrated success in reducing mortality, morbidity and hospital length of stay (LOS) (4). A critical element of ERAS programs is perioperative and postoperative analgesia. Not only does effectively administered analgesia enable greater patient satisfaction by minimising acute postoperative pain, but through alleviating the acute physiological stress response to surgery it facilitates enhanced recovery outcomes including early mobilisation and return to normal activity, reduced length of hospital stay, and re- duced cardiac, respiratory and gastrointestinal postoperative complications leading to improved long-term patient survival rates (5).

The optimal analgesic regime for surgical procedures is a critical clinical question that remains under ceaseless debate as the field of anaesthesia evolves. A multimodal approach combining regional anaesthesia, centrally-acting analgesics such as paracetamol, and drugs with a peripheral nonsteroidal anti-inflammatory effect is recommended to minimise the opioid-related side effects while maintaining an effective level of pain control (6). Epidural analgesia has been considered the cornerstone analgesic modality for major surgeries due to its ability to provide superior postoperative pain control (as measured by visual analogue pain scores at rest and on movement) compared to intravenous opioid administration (7). This is regardless of the type of surgery, type and time of pain assessment, analgesic agent, and epidural regimen $(8,9)$. It has been recognised that excellent postoperative pain control alle- 
viates the strain on intensive care unit resources, and an increasing trend in regional anaesthesia use has contributed to the ascent in the use of epidural analgesia in major surgeries (10).

In addition to improved analgesia, epidural analgesia is perceived to confer a whole host of additional benefits culminating in reduced postoperative morbidity. These include reduced incidence of cardiovascular, thromboembolic, and pulmonary complications, reduced incidence of postoperative ileus, and reduced requirement for additional opioid analgesia (11). The proposed mechanism by which epidural analgesia achieves these therapeutic outcomes is by mitigating the surgical stress and autonomic reflex responses following major surgeries (12). More specifically, it is postulated that epidural analgesia alleviates the catecholamine surge leading to increased cardiovascular workload and myocardial oxygen requirements, in addition to the hypercoaguable state following major surgery $(13,14)$. Furthermore physiological disturbances impairing pulmonary function are minimised, and gastrointestinal motility is preserved by infusion of local anaesthetics into the thoracic epidural space as sympathetic stimulation is blocked at the same time as parasympathetic innervation is maintained $(15,16)$.

Accordingly a meta-analysis of randomised studies conducted by Rodgers and colleagues in 2000 involved 141 trials and a total of 9559 patients, and found that neuraxial blockade reduced the likelihood of respiratory depression by $59 \%$, pulmonary embolism by $55 \%$, deep vein thrombosis by $44 \%$, pneumonia by $39 \%$, and myocardial infarction by $33 \%$ (all $\mathrm{P}<0.001$ ) (8). However this study examined primarily single-dose epidural protocols in major orthopaedic surgeries. In contrast, the standard protocol for epidural analgesia in major HPB surgery is continuous infusion of local anaesthetic at the thoracic level. The stress response mitigation associated with epidural analgesia is more marked in lower body procedures than in major abdominal and thoracic procedures (17).

A large international and multicenter landmark randomised controlled trial (RCT) found that combined intraoperative epidural and general anaesthesia in addition to postoperative epidural analgesia did not significantly reduce the incidence of most complications in high-risk patients undergoing major abdominal surgery (7). The RCT found no significant statistical difference in the incidence of major cardiovascular complications or postoperative mortality, but determined that respiratory failure was significantly reduced by epidural analgesia ( $\mathrm{P}=$ 0.02). Correspondingly, a 2004 review of epidural analgesia in gastrointestinal surgery by Fotiadis et al. concurred that epidural analgesia does not reduce the incidence of thromboembolism or cardiac morbidity in gastrointesti- nal surgery, but is associated with fewer respiratory complications, reduced duration of postoperative ileus and decreased hospital costs (16). Given the variability between studies in epidural regimens used, surgical operations examined, and conditions under which research was conducted, it is difficult to draw a definite conclusion on what post-operative morbidity benefits epidural analgesia confers.

Despite its popularity, epidural analgesia is not without its critics. In addition to doubts regarding its benefit on post-surgical morbidity and mortality, there exist concerns on complication risk especially since epidural is an invasive, high-cost, labour-intensive technique. Incidence of hypotension and bradycardia has been demonstrated to be increased following epidural anaesthesia (18). It has been suggested that continuous infusion of epidural analgesia may compromise pancreatic-enteric anastomotic healing and raise the risk of anastomotic leak (19). Furthermore epidural anaesthesia presents with an increased risk of epidural hematoma formation - a rare but fatal complication - secondary to coagulation changes after HPB surgery (20). This risk is present even in patients with normal preoperative coagulation undergoing uncomplicated hepatectomy (21). It is critical to remove the epidural catheter in a timely manner to minimize postoperative coagulation disturbances (22). Hence despite its proven analgesic efficacy, epidural analgesia may not have the optimal safety profile for patients undergoing major HPB surgery.

Multiple studies have highlighted high rates of epidural failure and epidural-related complications in pancreaticoduodenectomy patients - an important subclass of patients undergoing major HPB surgery. A retrospective observational study conducted at a high-volume surgical centre in Melbourne, Australia examined epidural failure rates and complications in 150 patients undergoing pancreaticoduodenectomy between 2004 - 2012, and determined a high epidural failure rate (conversion to opioid analgesia, or epidural related complication) of $63 \%$ (23). Given the technically challenging nature of major open HPB surgeries such as pancreaticoduodenectomy and the poor health profile of patients undergoing this type of surgery - generally being of elderly age and afflicted with multiple comorbidities - such a prominent adverse effect profile for epidural analgesia is unacceptable.

Given the concerns raised with epidural analgesia in the context of major open HPB surgery, intrathecal or spinal anaesthesia has emerged as a promising best practice yielding better patient outcomes. Intrathecal anaesthesia is a simpler and quicker alternative neuraxial anaesthesia technique, with a lower rate of technical failure (6). A meta-analysis by Meylan et al. assessing 645 patients over 
twenty-seven studies found that intrathecal morphine reduces pain at rest and on movement on the first postoperative day after major abdominal surgery with low complication risk (24).

Additionally, intra-operative and post-operative opioid requirements were found to be significantly reduced up to 48 hours after surgery. The primary concern with intrathecal morphine is the increased risk of postoperative respiratory depression (25). Consequently patients who have been managed with intrathecal opioid analgesia require close monitoring in an post-anaesthetic care unit (PACU) or intensive care unit (ICU) post-operation, and naloxone is administered to reverse opioid-induced respiratory depression. Accordingly a growing number of hospitals worldwide have adopted intrathecal morphine as a first choice perioperative analgesia for major open hepato-pancreaticbiliary surgery (26). Nonetheless the debate continues as to the optimal analgesic modality for major hepatopancreatic-biliary surgery.

This review aims to systematically evaluate the literature comparing intrathecal morphine analgesia to other analgesic modalities following major open hepatopancreatic-biliary surgery and assess the effects on analgesia and postoperative complications. These are chief outcomes in evaluating the success of an analgesic modality.

\section{Evidence Acquisition}

This review was structured with reference to the PRISMA guidelines (27). Relevant articles published over a 18-year period between January 2000 and March 2018 were found via searching the electronic databases EmBase, Medline and Cochrane Database of Systematic Reviews. Studies published before this period were not included as we deemed these studies to be out-of-date with contemporary practices. The following search terms were used: "liver resection" or "hepatic resection" or "hepatectomy" or "pancreas surgery" or "biliary tract surgery" and "analgesia" or "analgesic" or "intrathecal" or "spinal" or "epidural" or "opioid" or "opiate" and "postoperative pain". Additional relevant studies were discovered through bibliographies of retrieved articles. The specific search strategy is outlined in Supplementary File Appendix 1.

The two authors independently conducted the literature search and included all relevant articles. EndNote X7 was used to aid in the selection process. The authors independently reviewed the title and abstracts to determine eligibility of the paper for further assessment through fulltext reading with reference to the predetermined inclusion criteria. The following data were extracted directly from the papers: publication year, country, time period in which subjects underwent the intervention, number of subjects, procedure, analgesic protocol, patient demographic details, complication rates, inpatient mortality rates, length of stay (LOS), and pain scores on movement and at rest at 24-hour postoperation.

\subsection{Protocol Registration}

This review was registered on the PROSPERO international prospective register of systematic reviews on 28 February 2018 under the registration number CRD42018089726.

\subsection{Quality Assessment}

The Downs and Black method was used to assess the methodological quality of each included paper (28). This quality assessment tool is designed for assessing both randomised and non-randomised studies. Question 27 was altered to "Was a power analysis done?" with a score of 1 assigned to "yes" and a score of 0 assigned to "no". One reviewer assessed study quality using a data-extraction form. Studies were given a modified Downs and Black score out of maximum attainable value of 27 .

\subsection{Inclusion and Exclusion Criteria}

Clinical trials assessing spinal analgesia in open major open hepato-biliary-pancreatic surgery were included. Papers examining analgesia in laparoscopic surgery were excluded. Papers not written in English and previously reported systematic reviews were excluded. Papers comparing alternative arrangements of a single analgesic technique were excluded. Conference abstracts were excluded.

\subsection{Outcome}

The primary outcome was pain scores at 24 hours at rest and on movement. Secondary outcomes were postoperative opioid consumption within 72 postoperative hours, length of stay (LOS), intra-operative fluid administration and post-operative fluid administration within 72 postoperative hours, and complication rate within 30 postoperative days. All medical and surgical complications were included.

\subsection{Analysis}

A qualitative descriptive analysis was performed to compare outcomes among the selected trials and discover statistically significant differences. A statistically significant difference was defined as having a P value of $\leq 0.05$. 


\section{Results}

The search returned 815 studies after the removal of duplicates. Titles and abstracts were screened for relevance, and 28 full-text articles that met the inclusion criteria were retrieved. Eighteen of these studies were excluded after full manuscript review: sixteen studies were excluded because they discussed laparoscopic surgery, one study was excluded because both study arms involved intrathecal opioid administration, one study was excluded because it was a conference abstract. The study selection process is outlined in Figure 1.

\subsection{Study Characteristics}

Ten studies were included in the review $(26,29-37)$ as shown in the PRISMA diagram (Figure 1). Six of the studies were RCTs (29-31, 33, 35, 37), two studies were retrospective observational studies $(26,34)$, and two studies were prospective observational studies $(32,36)$. The selected articles reached a median modified Down's and Black score of 22.5 out of 27 (range $16-26$ ).

All studies included adult patients only. The studies were published between 2002 and 2016. The studies were performed in eight countries: New Zealand (two trials), South Korea (two trials), UK (one trial), Iran (one trial), France (one trial), Australia (one trial), Canada (one trial) and Italy (one trial). Study sizes ranged from 40 to 161 patients. A total of 663 patients were included in trials.

Five different analgesic methods were assessed in comparison to intrathecal analgesia: epidural analgesia, placebo, patient-controlled analgesia, intravenous opioid, and continuous wound infiltration. No more than one RCT for each analgesic regimen assessed against intrathecal morphine was available, thus meta-analysis was unfeasible. Study characteristics are outlined in Table 1. Analgesic protocols in each study are represented in Table 2. Outcomes are shown in Tables 3 - 5.

\subsection{Intrathecal (ITM) Versus Epidural}

Five studies compared ITM to epidural analgesia. Of these, four were observational studies and one was a randomised, controlled trial.

Four studies reported on pain scores in the postoperative period $(30,32,34,36)$. Three found that intrathecal morphine provided superior analgesia at certain periods within 72-hours after surgery. Koea et al.'s study (34) measured pain scores for five days post-operation, and found that pain scores were similar between ITM and epidural groups except on postoperative day 3 (ITM 0 vs. epidural $4, \mathrm{P}=0.05$ ), when the epidural group described significantly higher pain scores on postoperative day 3 following weaning of epidural analgesia. Kasivisvanathan et al.'s study (36) demonstrated that patients treated with ITM + fPCA had reduced pain scores for the first 24-hour after surgery $(\mathrm{P}<0.010)$ compared to the epidural group, after which there was no significant difference. Duncan's study found that pain scores were consistently lower in the intrathecal group on postoperative days one, two and three. Duncan et al.'s study (32) did not indicate whether the difference between the ITM and epidural group pain scores were statistically significant. In contrast De Pietri et al.'s study (30) determined that ITM did not provide inferior analgesia to continuous epidural analgesia up to 48 hours post operation.

Four studies reported on post-operative rescue analgesic requirements $(26,30,34,36)$. Three $(26,30,36)$ found that patients treated with ITM required rescue analgesia more often, and in higher doses. Sakowska et al.'s study (26) determined that a higher proportion of patients in the intrathecal group required additional analgesia in ICU (ITM 68\% vs. TEA 16\%, P < 0.001). Kasivisvanathan et al.'s study (36) determined that patients treated with intrathecal morphine had a higher cumulative IV fentanyl use in the first 4 post-operative days (ITM + fPCA $532 \mu \mathrm{g}$ vs. epidural $119 \mu \mathrm{g}$, values are medians, $\mathrm{P}<0.001)$. De Pietri et al.'s (30) study determined that patients treated with ITM used more PCA morphine in the first 48-hours after surgery (mean cumulative PCA morphine use - ITM 12.1 $\mu \mathrm{g}$ vs. epidural $3.1 \mu \mathrm{g}, \mathrm{P}<0.01)$. In contrast Koea et al.'s study (34) found that patients treated with epidural analgesia required higher doses of oral opioid than patients treated with intrathecal morphine on postoperative days three (median daily dose oral opioid ITM 0 mg vs. epidural $100 \mathrm{mg}, \mathrm{P}<0.05$ ) and four (median daily dose oral opioid ITM 0 mg vs. epidural $85 \mathrm{mg}, \mathrm{P}<0.05)$.

Four studies $(26,32,34,36)$ assessed length of stay (LOS) and each independently found that LOS was significantly longer in patients treated with epidural analgesia.

Three studies $(26,34,36)$ evaluated intraoperative fluid administration and each independently found that patients treated with ITM required less intraoperative fluid than patients treated with epidural analgesia.

Four studies $(26,32,34,36)$ evaluated major complication rates. Sakowska et al., Kasivisvanathan et al., and Koea et al. determined no significant difference between patients treated with intrathecal morphine and patients treated with epidural analgesia (See Table 5).

Three studies $(26,32,36)$ reported failed epidurals: Sakowska's trial found 3 failed epidurals, Kasivisvanathan et al.'s study found 7 failed epidurals, and Duncan's study found 1 failed intrathecal and 5 failed epidurals.

Koea et al.'s and Kasivisvanathan et al.'s studies (34, 36) determined no significant difference in incidence of major complications between patients managed with in- 


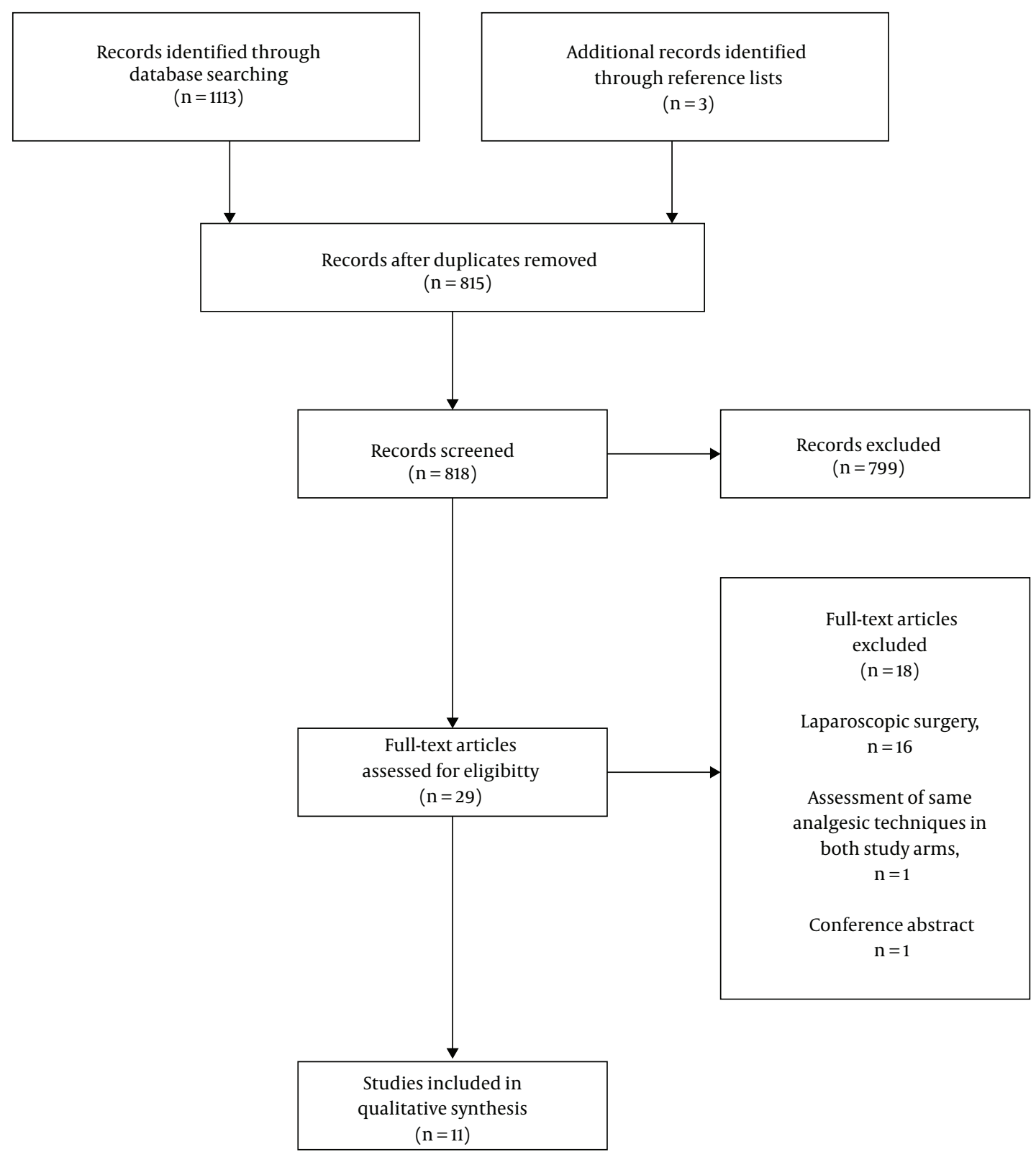

Figure 1. PRISMA flow chart depicting study selection

trathecal morphine and patients managed with epidural anaglesia. Sakowska et al.'s study (26) determined that patients managed with epidural analgesia had a significantly higher incidence of respiratory complications and postoperative hypotension.

In terms of minor complications, De Pietri's study (30) found that patients treated with intrathecal had a higher rate of vomiting (ITM $16 \%$ vs. TEA $4 \%, \mathrm{P}<0.05$ ) and pruritis (ITM $16 \%$ vs. TEA 0\%, P < 0.05)
3.3. Intrathecal (ITM) Versus Patient-Controlled Analgesia (PCA)

Two randomised controlled trials assessed ITM combined with PCA, compared to PCA alone $(29,33)$. One trial assessed PCA morphine, and the other trial assessed PCA fentanyl. Both Ko et al.'s (33) and Devys et al.'s (29) trials found that patients treated with intrathecal morphine had significantly lower pain scores as measured by the VAS within the first 24 hours post-operation. Devys et 


\begin{tabular}{|c|c|c|c|c|c|c|}
\hline $\begin{array}{l}\text { First Author, Year of } \\
\text { Publication }\end{array}$ & Country & Number of Subjects & Design & \multicolumn{2}{|c|}{ Operation } & $\begin{array}{c}\text { Modified Downs and Black } \\
\text { Score } \\
\end{array}$ \\
\hline Sakowska, 2009 (26) & New Zealand & 161 & Retrospective cohort study & \multicolumn{2}{|c|}{ Hepatobiliary surgery } & $21 / 27$ \\
\hline Roy, 2006 (31) & Canada & 40 & RCT & \multicolumn{2}{|c|}{ Liver resection } & $26 / 27$ \\
\hline Kasivisvanathan, 2014 (36) & UK & 73 & Prospective cohort study & \multicolumn{2}{|c|}{ Liver resection } & $22 / 27$ \\
\hline Koea, 2009 (34) & New Zealand & 100 & Retrospective cohort study & \multicolumn{2}{|c|}{ Liver resection } & $21 / 27$ \\
\hline De Pietri, 2006 (30) & Italy & 50 & RCT & \multicolumn{2}{|c|}{ Liver resection } & $24 / 27$ \\
\hline Devys, $2003(29)$ & France & 60 & RCT & \multicolumn{2}{|c|}{ Major abdominal surgery } & $26 / 27$ \\
\hline Ко, 2009 (33) & South Korea & 40 & RCT & \multicolumn{2}{|c|}{ Donor right hepatectomy } & $22 / 27$ \\
\hline Lee, $2013(35)$ & South Korea & 45 & RCT & \multicolumn{2}{|c|}{ Donor right hepatectomy } & $26 / 27$ \\
\hline Dichtwald, 2017(37) & Iran & 49 & RCT & \multicolumn{2}{|c|}{ Liver or pancreas resection } & $23 / 27$ \\
\hline Duncan, $2007(32)$ & Australia & 45 & Prospective cohort study & \multicolumn{2}{|c|}{ Major abdominal surgery } & $16 / 27$ \\
\hline \multicolumn{7}{|c|}{ Table 2. Study Analgesic Regimens } \\
\hline $\begin{array}{l}\text { First author, Year of } \\
\text { Publication }\end{array}$ & Modality 1 & Regimen & & Modality 2 & Regim & \\
\hline Sakowska, 2009 (26) & Intrathecal morphine & $\begin{array}{l}\text { Doses (morphine } \\
\text { bupivacaine) left } \\
\text { the individual an }\end{array}$ & $\begin{array}{l} \pm \text { fentanyl } \pm \\
o \text { the discretion of } \\
\text { esthetist }\end{array}$ & Epidural & $\begin{array}{l}0.2 \% \mathrm{rc} \\
\text { rate tai }\end{array}$ & $\begin{array}{l}\text { ivacaine }+ \text { fentanyl } 2 \mu \mathrm{g} / \mathrm{mL} \text {; } \\
\text { red by acute pain team }\end{array}$ \\
\hline Roy, 2006 (31) & Intrathecal morphine & $500 \mu \mathrm{g}$ morphin & $+15 \mu$ g fentanyl & Placebo & Sham i & rathecal \\
\hline $\begin{array}{l}\text { Kasivisvanathan, } \\
2014 \text { (36) }\end{array}$ & $\begin{array}{l}\text { Intrathecal morphine + } \\
\text { patient controlled } \\
\text { analgesia }\end{array}$ & $\begin{array}{l}5 \mu \mathrm{g} / \mathrm{kg} \text { of intratl } \\
\text { (maximum dose } \\
\text { of } 0.5 \% \text { heavy bu } \\
\text { fentanyl } 25 \mu \mathrm{g} \text { bc } \\
\text { four }\end{array}$ & $\begin{array}{l}\text { ecal morphine } \\
00 \mu \mathrm{g})+2.5-3.0 \mathrm{~mL} \\
\text { ivacaine; PCA } \\
\text { us ceased by POD }\end{array}$ & Epidural & $\begin{array}{l}\mathrm{T} 7-\mathrm{Tg} \\
\mu \mathrm{g} / \mathrm{mL} \\
\text { of } 5-10\end{array}$ & $\begin{array}{l}\text { 125\% bupivacaine } 7-10 \mathrm{~mL}+2 \\
\text { entanyl; continuous infusion } \\
\mathrm{nL} / \mathrm{h} \text {; weaned after } 72 \text { hours }\end{array}$ \\
\hline Koea, $2009(34)$ & Intrathecal morphine & $\begin{array}{l}300 \mu \mathrm{g} \text { morphin } \\
\text { (1200 mg preop a }\end{array}$ & $\begin{array}{l}\text { + oral gabapentin } \\
\text { id } 400 \text { mg bd postop) }\end{array}$ & Epidural & $\begin{array}{l}0.125 \% \\
0.1 \mathrm{~mL} /\end{array}$ & $\begin{array}{l}\text { upivacaine + fentanyl } 2 \mu \mathrm{g} / \mathrm{mL} \text {; } \\
\text { s/h weaned after } 72 \text { hours }\end{array}$ \\
\hline De Pietri, 2006 (30) & Intrathecal morphine & L3 - L5 $200 \mu \mathrm{g} \mathrm{mo}$ & phine & Epidural & $\begin{array}{l}\text { T9-T11 } \\
\text { morph } \\
\text { ropiva } \\
\text { infusio }\end{array}$ & $\begin{array}{l}2 \% \text { ropivacaine } 6-8 \mathrm{~mL} \text { and } \\
\text { e } 2 \mathrm{mg} \text { bolus preop; } 0.2 \% \\
\text { ine } 5-7 \mathrm{~mL} / \mathrm{h} \text { continuous }\end{array}$ \\
\hline Devys, 2003 (29) & $\begin{array}{l}\text { Intrathecal morphine + } \\
\text { patient controlled } \\
\text { analgesia }\end{array}$ & $\begin{array}{l}\text { L3-4 } 300 \mu \mathrm{g} \text { or } 40 \\
\text { PCA delivering } 1 \mathrm{r} \\
\text { with five-minute }\end{array}$ & $\begin{array}{l}\mu \mathrm{g} \text { morphine }+\mathrm{IV} \\
\text { g morphine boluses } \\
\text { ock-out interval }\end{array}$ & $\begin{array}{l}\text { Patient controlled } \\
\text { analgesia }\end{array}$ & $\begin{array}{l}\text { Up to } 2 \\
\text { pain co } \\
\text { mg mo } \\
\text { lock-ol }\end{array}$ & $\begin{array}{l}\text { mg morphine titration on first } \\
\text { plaint + IV PCA delivering } 1 \\
\text { phine boluses with five-minute } \\
\text { interval }\end{array}$ \\
\hline Ко, 2009 (33) & $\begin{array}{l}\text { Intrathecal morphine + } \\
\text { patient controlled } \\
\text { analgesia }\end{array}$ & $\begin{array}{l}\text { L3-5 } 400 \mu \mathrm{g} \text { mor } \\
\text { fentanyl } 15 \mu \mathrm{g} \text { bo } \\
\text { min and baseline }\end{array}$ & $\begin{array}{l}\text { hine sulfate + } \\
\text { us, lockout time of } 15 \\
\text { rate of } 15 \mu \mathrm{g} / \mathrm{h}\end{array}$ & $\begin{array}{l}\text { Patient controlled } \\
\text { analgesia }\end{array}$ & $\begin{array}{l}\text { Fentan } \\
\text { min an }\end{array}$ & $\begin{array}{l}15 \mu \mathrm{g} \text { bolus, lockout time of } 15 \\
\text { baseline rate of } 15 \mu \mathrm{g} / \mathrm{h}\end{array}$ \\
\hline Lee, $2013(35)$ & Intrathecal morphine & $\begin{array}{l}\mathrm{L} 3-5400 \mu \mathrm{g} \mathrm{mor} \\
\text { fentanyl in } 100 \mathrm{~m} \\
\text { delivering } 1 \mathrm{~mL} / \mathrm{h}\end{array}$ & $\begin{array}{l}\text { hine }+1500 \mu \mathrm{g} \text { of } \\
\text { of normal saline } \\
\text { ur }\end{array}$ & $\begin{array}{l}\text { Continuous wound } \\
\text { infusion }\end{array}$ & $\begin{array}{l}0.75 \% \mathrm{r} \\
\text { ropivo } \\
300 \mathrm{ml} \\
\text { consta } \\
\text { from e }\end{array}$ & $\begin{array}{l}\text { ivocaine } 10 \mathrm{ml} \text { bolus }+ \\
\text { ine wound infusion delivering } \\
\text { f } 0.5 \% \text { ropivacaine at a } \\
\text { rate of } 4 \mathrm{~mL} / \text { hour for } 72 \text { hours } \\
\text { of operation }\end{array}$ \\
\hline Dichtwald, 2017(37) & Intrathecal morphine & L3-5 $4 \mu \mathrm{g} / \mathrm{kg}$ mor & hine & Intravenous opioid & $\begin{array}{l}0.1-0.2 \\
\text { infusio } \\
\text { bolus } \\
\text { the enc }\end{array}$ & $\begin{array}{l}\mathrm{gg} / \mathrm{kg} / \mathrm{min} \text { remifentanil } \\
\text { during surgery followed by IV } \\
\text { morphine, } 0.15 \mathrm{mg} / \mathrm{kg} \text { before } \\
\text { f surgery }\end{array}$ \\
\hline Duncan, 2007 (32) & Intrathecal morphine & $\begin{array}{l}\text { (Morphine } 10 \mu \mathrm{g} \\
\mu \mathrm{g} / \mathrm{mL}+0.05 \% \mathrm{~b} \\
\text { commenced at } 2\end{array}$ & $\begin{array}{l}\mathrm{nL}+\text { midazolam } 100 \\
\text { pivacaine) } \\
\mathrm{nL} / \mathrm{h} \text {. }\end{array}$ & Epidural & $\begin{array}{l}0.125 \% \\
\text { at } 6 \text { to }\end{array}$ & $\begin{array}{l}\text { upivacaine + fentanyl } 2 \mu \mathrm{g} / \mathrm{mL} \\
\mathrm{ml} / \mathrm{h} \text {. }\end{array}$ \\
\hline
\end{tabular}

al.' study determined that patients treated with intrathecal morphine required less PCA morphine in the first 24 hours post-operation (ITM + PCA $9 \mathrm{mg}$ vs. PCA $40 \mathrm{mg}, \mathrm{P}=$ 0.0001). Similarly, Ko et al.'s study determined the ITM + PCA group required less intraoperative remifentanil (ITM
+ PCA: $5760.2 \pm 1582.3$ mg vs. PCA: $9496.6 \pm 3092.2, \mathrm{P}<$ $0.05)$ and less supplementary postoperative PCA fentanyl (ITM + PCA: 1314 mg vs. PCA: 1500, P $<0.05)$. In terms of minor complications, Devys et al.'s study determined a significantly increased incidence of nausea and vomiting in the 


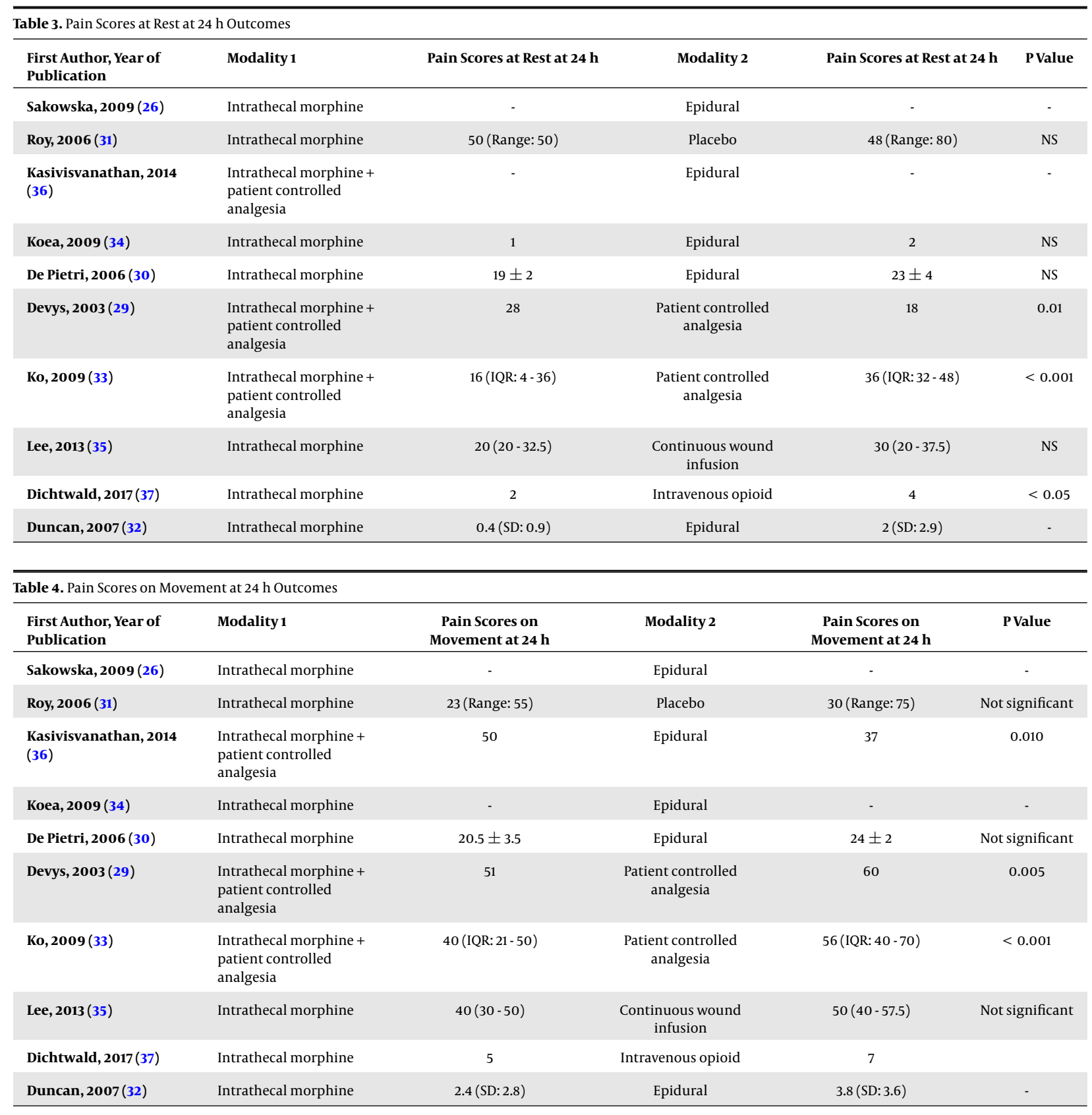

ITM group 24-hours after surgery (ITM + PCA: 53\% vs. PCA: $23 \%, P=0.016)$, and Ko et al.'s study determined a significant increased incidence of pruritis in the ITM group 24-hours after surgery $(55 \%, \mathrm{P}<0.001)$. There were no other reported difference in complications rates in either study.

\subsection{Intrathecal (ITM) Versus Placebo}

One randomised controlled trial assessed ITM compared with a placebo (sham intrathecal) (31). This study conducted by Ko et al. found significantly improved pain scores at rest and on movement up to 18-hours postoperation in the ITM group, as well as significantly reduced cumulative morphine consumption at 48-hours post-operation ( $47 \pm 21 \mathrm{mg}$ vs. $124 \pm 30 \mathrm{mg}, \mathrm{P}<0.0001$ ). The study in fact was stopped midway after 20 patients were enrolled due to the large difference in morphine consumed between the treatment and control group. This was the only trial reviewed which was stopped before completion. Ko et al. also found no significant difference in complication rates between the study arms, however only seda- 


\begin{tabular}{|c|c|}
\hline $\begin{array}{l}\text { First Author, Year of } \\
\text { Publication }\end{array}$ & Complications \\
\hline Sakowska, 2009 (26) & $\begin{array}{l}\text { There was a significantly higher incidence of respiratory complications (epidural anaesthesia } 10 \% \text { vs. intrathecal morphine } 1 \%, \mathrm{P}= \\
0.02 \text { ) and postoperative hypotension (epidural anaesthesia } 41 \% \text { vs. intrathecal morphine } 9 \%, \mathrm{P}<0.001 \text { ) in the epidural group. There } \\
\text { were three cases of respiratory depression, all from the intrathecal morphine group. There were } 19 \text { failed epidurals. }\end{array}$ \\
\hline Roy, $2006(31)$ & No significant difference found in the incidence of sedation, nausea or pruritis. \\
\hline Kasivisvanathan, 2014 (36) & $\begin{array}{l}\text { No significant difference was found in complication rates. There were } 7 \text { failed epidurals, from which two cases of respiratory } \\
\text { depression emerged requiring medical intervention. }\end{array}$ \\
\hline Koea, 2009 (34) & No significant difference was found in complication rates. \\
\hline De Pietri, 2006 (30) & $\begin{array}{l}\text { Patients treated with intrathecal had a higher rate of vomiting (intrathecal morphine } 16 \% \text { vs. epidural } 4 \%, \mathrm{P}<0.05 \text { ) and pruritis } \\
\text { (intrathecal morphine } 16 \% \text { vs. epidural } 0 \%, \mathrm{P}<0.05 \text { ). }\end{array}$ \\
\hline Devys, $2003(29)$ & $\begin{array}{l}\text { Patients treated with intrathecal morphine had a higher incidence of nausea and vomiting within } 24 \text { hours post-operation } \\
\text { (intrathecal morphine + patient controlled analgesia } 53 \% \text { vs. patient controlled analgesia } 23 \%, \mathrm{P}=0.016 \text { ). }\end{array}$ \\
\hline Ko, $2009(33)$ & No significant difference was found in complication rates. \\
\hline Lee, $2013(35)$ & No significant difference was found in complication rates. \\
\hline Dichtwald, 2017 (37) & No significant difference found in the incidence of sedation, nausea or pruritis. \\
\hline Duncan, 2007 (32) & 5 cases of unintentional epidural termination. 1 case of unintentional intrathecal morphine termination. \\
\hline
\end{tabular}

tion, nausea and pruritus were assessed.

\subsection{Intrathecal (ITM) Versus Continuous Wound Infusion (CWI)}

One randomised controlled trial assessed ITM compared with CWI (35). This study by Lee et al. found that pain scores were significantly improved in the ITM group for the first 12-hours post-operation, following which they became statistically similar. CWI was found to have a faster time to first flatus (CWI $4308.4 \pm 646.4 \mathrm{mg}$ vs. ITM 4874.6 \pm 940.4 , $\mathrm{P}=0.03$ ), but despite this LOS was not reduced (CWI 17.9 \pm $11.6 \mathrm{mg}$ vs. ITM $15.0 \pm 7.2, \mathrm{P}=0.44)$. ITM was also associated with higher postoperative IV fentanyl consumption (CWI 915 vs. ITM 1500, P=0.004). There was no significant difference in complication rates between the two study arms.

\subsection{Intrathecal (ITM) Versus Intravenous Opioid (IVO)}

One randomised controlled trial assessed ITM compared with IVO (37). This study by Dichtwald et al. found higher (but not reaching statistical significance) intraoperative fluid requirements in the IVO group. Additionally ITM patients experienced lower pain scores and required less additional morphine(ITM $4.8 \pm 3.7 \mathrm{mg}$ vs. IVO $14.7 \pm 5.3$ $\mathrm{mg}, \mathrm{P}=0.03$ ). There was no significant difference in complication rates between the two study arms.

\subsection{Complications}

No study reported any incidences of neurological sequelae (postdural puncture headache or spinal hematoma). Only one study (26) reported deaths: (ITM 2 (3\%) vs. Epidural $2(4 \%), P=0.65)$. Three trials $(26,32,36)$ found a total of twenty-seven cases of failed epidurals and one case of failed intrathecal morphine. Two trials (26, 36) commented specifically on respiratory depression: There were three cases in the ITM group in Sakowska et al.'s study and two cases stemming from failed epidurals in Kasivisvanathan et al.'s study. Neither of these reached statistical significance.

\section{Discussion}

This review identified a wide range of international studies assessing intrathecal morphine against alternative analgesia modalities over a period of almost 15 years.

\subsection{Post-Operative Analgesia}

Eight studies (29-33, 35-37) assessed postoperative analgesia as measured by pain scores. Measured against placebo, CWI and PCA, patients treated with intrathecal morphine demonstrated significantly improved pain scores for up to the first 24 hours post-operation. Measured against IVO, patients treated with intrathecal morphine demonstrated significantly improved pain scores for the entire $72 \mathrm{~h}$ follow-up period. These results were all determined by randomised controlled trials. Hence our selected studies indicate that intrathecal morphine provides superior analgesia to alternative analgesia modalities (excluding epidural) for up to 24-hours after surgery.

The findings of intrathecal morphine compared to epidural were not as clear. One prospective cohort study (36) and one retrospective cohort study (26) with similar epidural analgesia and intrathecal analgesia regimens determined that intrathecal morphine provided superior 
analgesia for certain periods in the 72-hours after surgery. The one randomised controlled trial conducted by De Pietri et al. (30) determined that intrathecal morphine did not provide inferior analgesia to epidural. While each trial differed in their outcomes, the tentative suggestion is that single-shot intrathecal morphine provides at least similar analgesia to continuous epidural analgesia.

Overall our studies suggest that single-dose intrathecal morphine provides the best available analgesia for acute postoperative pain.

\subsection{Need for Rescue Analgesia}

Nine studies (26, 29-31,33-37) assessed requirements for rescue analgesia post-operation. Patients treated with intrathecal morphine required were shown to require less rescue analgesia than patients treated with intravenous opioid or PCA only $(29,37)$. On the other hand patients treated with intrathecal morphine were shown to require more rescue analgesia than patients treated with CWI, as three of the four studies $(26,30,36)$ assessing intrathecal analgesia against epidural analgesia mirrored this finding.

Our studies suggest that single-dose intrathecal morphine requires higher amounts of rescue analgesia than epidural or CWI. Therefore despite intrathecal morphine providing the lowest pain scores in our studies, with its heightened requirement for postoperative pain relief it cannot be concluded that intrathecal morphine has the greatest analgesic potency of all regimens examined.

\subsection{Fluid Administration and Postoperative Hypotension}

Six studies $(26,33-37)$ assessed fluid administration. No significant difference was found in fluid administration between patients administered intrathecal morphine, and those administrated PCA, CWI or IVO. However all three studies $(26,34,36)$ assessing fluid administration between intrathecal and epidural groups found that patients treated with epidural analgesia had significantly increased fluid administration than patients treated with intrathecal morphine. Increased fluid administration in patients treated with epidural analgesia has been thought to be associated with the increased incidence of postoperative hypotension secondary to epidural-induced sympathetic blockade (38). Accordingly Sakowska's study (26) found a significantly increased incidence of postoperative incidence in patients treated with epidural analgesia, and three patients in De Pietri et al.'s study (30) required increased fluid support to treat hypotension.

Intraoperatively, epidural-induced hypotension can be useful in maintaining low central venous pressure (CVP) during hepatic resection, which reduces blood loss and transfusion requirements (39). However postoperatively increased volumes of IV fluid and doses of vasopressor doses are required to circumvent hypotension. Hence epidural analgesia predisposes patients to the well-known perils of excessive IV fluid administration (40).

However a randomised controlled trial by Aloia et al. found that hypotension can be minimised in the context of epidural anaesthesia use in major open hepatobiliary surgery via administration of lower-than-standard concentration of local anaesthetic ( $0.075 \%$ bupivacaine), and implementation of a regimented rescue bolus schedule (41). In summary, epidural analgesia is associated with a risk of postoperative hypotension and hence fluid overload that is not present with intrathecal analgesia, but this risk can be mitigated with adherence to the correct protocol.

\subsection{Length of Stay}

Seven studies $(26,32-37)$ assessed length of stay. The included studies demonstrated no difference in LOS between patients treated with intrathecal morphine, and patients treated with PCA, CWI or IVO. However all four studies measuring LOS in epidural groups against intrathecal groups found that patients treated with intrathecal morphine had a shorter length of stay than patients treated with continuous epidural infusion.

This can be accounted for by patients treated with intrathecal morphine requiring a lower amount of intravenous fluid therapy, thereby enabling an earlier urinary catheter removal and hence earlier time to mobilization (34).

\subsection{Complications}

Intrathecal morphine was not correlated with an increased risk of major complications in the intra-operative or postoperative period compared to other analgesic modalities. With respect to minor complications, both De Pietri et al.'s (30) and Devys et al.' trials (29) determined an increased incidence of pruritis and nausea in patients treated with intrathecal morphine. These more common side effects are not life threatening and managed with ease (42).

Across the selected studies, a total of twenty-seven failed epidurals and one failed intrathecal was reported. The evidence is substantial that the failure rate of epidural analgesia is significantly higher than intrathecal analgesia (43). The epidural failure rate is especially heightened in major operations such as pancreaticoduodenectomy, as Pratt et al.'s study demonstrates (44). Pratt et al. found that these high epidural failure rates were due to insufficient analgesia and/or hemodynamic instability, and these patients necessitated more aggressive fluid resuscitation, thereby incurring increased rate of respiratory and gastrointestinal complications. 
Pratt also found that patients administered epidural anaesthetics perioperatively for pancreaticoduodenectomy significantly higher rates of major complications, than patients who did not receive epidural anaesthetics (44). Within Pratt et al.'s study, patients undergoing epidural anaesthesia more frequently encountered postoperative ileus and pancreatic fistulae, consequently requiring discharge to rehabilitation facilities more frequently. Pratt proposes that these findings can be accounted for by the tendency for excessive blood loss and sudden fluid shifts during pancreaticoduodenectomy. Thus for patients undergoing major hepato-pancreatic-biliary surgery, continuous epidural analgesia is associated with medical risks that are severe and not necessarily infrequent.

The major safety concern with intrathecal morphine is respiratory depression. Within the review's included trials, only Sakowska et al.'s (26) reported incidences of respiratory depression within patients administered intrathecal morphine. However there was no statistically significant difference between the ITM and epidural group in the incidence of respiratory depression.

A meta-analysis comparing ITM doses of $<300 \mu \mathrm{g}$, $>$ $300 \mu \mathrm{g}$ and placebo in a range of procedures found that there was a greater risk of respiratory depression with the higher dose group (9\%) when compared to systemic opioids (2\%), but not in the lower dose group (1\%) (45). Thus the risk of opioid-induced respiratory depression can be minimised by using the lowest effective dose of intrathecal morphine and keeping the patient under close surveillance for at least 18-24 hours following a single dose $(6,46)$.

It is suggested that intrathecal morphine - with its controllable risks of pruritus, nausea and respiratory depression - provides a much improved safety profile to continuous epidural analgesia - which suffers risks of postoperative hypotension, failed epidural, and lengthened time to mobilisation and length of stay among other medical risks.

\subsection{Quality and Methodological Limitations}

The analgesic regimens, healthcare settings and outcomes measured varied largely amongst the selected papers. While all included trials assessed intrathecal morphine administration, each trial prescribed a different dose of intrathecal morphine and differed in the supplementary analgesic specified ranging from fentanyl, bupivacaine, gabapentin and midazolam. In contrast, trials assessing epidural analgesia involved similar regimes for epidural analgesia - a continuous infusion of $0.125 \%$ bupivacaine or $0.2 \%$ ropivocaine added to fentanyl $2 \mu \mathrm{g} / \mathrm{mL}$ into the thoracic epidural space.

While nine of the trials assessed single-shot intrathecal morphine, Duncan et al.'s trial (32) was the only trial that assessed continuous intrathecal morphine infusion in the context of major abdominal surgery determined that intrathecal infusion. Furthermore this study did not use appropriate statistical techniques and included abdominal surgeries outside of major hepato-pancreatic-biliary surgeries. Therefore the findings of Duncan's trial have limited generalisability compared to our other selected studies.

Heterogeneity existed in outcomes recorded in each trial. Some studies did not capture data on pain scores and other studies did not capture data on major systemic complications, focusing only on minor outcomes such as pruritis, sedation and nausea. Furthermore certain outcomes could be measured in multiple ways - rescue analgesia consumption was either measured in percentage requiring rescue analgesia, or total PCA morphine or fentanyl administered in a certain postoperative period. The only outcome parameter which remained constant across different trials was length of stay (LOS). Each selected study was small, single-centre trial - the largest trial involved 161 patients. The hospital protocols varied from trial to trial, as did the patients. There were three subclasses of surgical operations conducted within the selected studies major hepato-biliary-pancreatic surgery (primarily hepatectomy), major abdominal surgery, and right liver donor hepatectomy. For these reasons we acknowledge that the comparing the primary and secondary outcomes between different trials each with unique and differing research methodologies is challenging and limits the findings of our review.

Large heterogeneity between selected studies, coupled with the fact that only one RCT was selected per analgesic protocol pair, prevented meta-analysis or definitive recommendations.

More randomised controlled trials are needed to evaluate intrathecal morphine against alternative analgesic modality, especially continuous epidural infusion. We recommend conducting a large multi-centre trial to further investigate the relative advantages and disadvantages of intrathecal morphine as an anaesthetic modality.

\subsection{Conclusions}

Based on the limited number of trials discovered, this review suggests that single-shot intrathecal morphine may be more appropriate than epidural analgesia as a first-line analgesic technique for major open hepatopancreatic-biliary surgery. While patients administered intrathecal morphine require larger doses of supplementary post-operative analgesia, they receive a non-inferior level of analgesia. Furthermore, these patients require less fluid therapy and hence have a shorter LOS. Medicals risks associated with single shot intrathecal morphine are controllable while continuous epidural analgesia is particularly 
dangerous in the setting of major open hepato-pancreaticbiliary surgery. However, to make definitive recommendations, large RCTs are required to substantiate this review's findings.

\section{Supplementary Material}

Supplementary material(s) is available here [To read supplementary materials, please refer to the journal website and open PDF/HTML].

\section{Footnotes}

Authors' Contribution: Jefferson Zhi Jie Tang and Laurence Weinberg designed the study, collected the data, and conducted the statistical analysis. Jefferson Zhi Jie Tang wrote the manuscript. Laurence Weinberg helped to write and revise the manuscript.

Conflict of Interests: There are no conflicts of interest for the author to declare.

Funding/Support: The authors declare that all funding was provided independently by the authors

\section{References}

1. Choo SP, Venook AP. Responding to the rising incidence of hepatocellular carcinoma with targeted therapy. Gastrointest Cancer Res. 2008;2(2):96-7. [PubMed: 19259302]. [PubMed Central: PMC2630824].

2. Ilic M, Ilic I. Epidemiology of pancreatic cancer. World J Gastroenterol. 2016;22(44):9694-705. doi: 10.3748/wjg.v22.i44.9694. [PubMed: 27956793]. [PubMed Central: PMC5124974].

3. Kneuertz PJ, Pitt HA, Bilimoria KY, Smiley JP, Cohen ME, Ko CY, et al. Risk of morbidity and mortality following hepato-pancreato-biliary surgery. J Gastrointest Surg. 2012;16(9):1727-35. doi: 10.1007/s11605-0121938-y. [PubMed: 22760965].

4. Hughes MJ, Ventham NT, McNally S, Harrison E, Wigmore S. Analgesia after open abdominal surgery in the setting of enhanced recovery surgery: A systematic review and meta-analysis. JAMA Surg. 2014;149(12):1224-30. doi: 10.1001/jamasurg.2014.210. [PubMed: 25317633].

5. Fawcett WJ, Baldini G. Optimal analgesia during major open and laparoscopic abdominal surgery. Anesthesiol Clin. 2015;33(1):65-78. doi: 10.1016/j.anclin.2014.11.005. [PubMed: 25701929].

6. Mugabure Bujedo B. A clinical approach to neuraxial morphine for the treatment of postoperative pain. Pain Res Treat. 2012;2012:612145. doi: 10.1155/2012/612145. [PubMed: 23002426]. [PubMed Central: PMC3395154].

7. Rigg JR, Jamrozik K, Myles PS, Silbert BS, Peyton PJ, Parsons RW, et al. Epidural anaesthesia and analgesia and outcome of major surgery: A randomised trial. Lancet. 2002;359(9314):1276-82. doi: 10.1016/S01406736(02)08266-1. [PubMed: 11965272].

8. Rodgers A, Walker N, Schug S, McKee A, Kehlet H, van Zundert A, et al. Reduction of postoperative mortality and morbidity with epidural or spinal anaesthesia: Results from overview of randomised trials. BMJ. 2000;321(7275):1493. doi:10.1136/bmj.321.7275.1493. [PubMed: 11118174]. [PubMed Central: PMC27550].

9. Wu CL, Cohen SR, Richman JM, Rowlingson AJ, Courpas GE, Cheung K, et al. Efficacy of postoperative patient-controlled and continuous infusion epidural analgesia versus intravenous patient-controlled analgesia with opioids: A meta-analysis. Anesthesiology. 2005;103(5):107988. quiz 1109-10. doi: 10.1097/00000542-200511000-00023. [PubMed: 16249683].

Anesth Pain Med. 2019; 9(6):e94441.
10. Wildsmith JA. Continuous thoracic epidural block for surgery: Gold standard or debased currency? Br J Anaesth. 2012;109(1):9-12. doi: 10.1093/bja/aes177. [PubMed: 22696554].

11. Nimmo SM, Harrington LS. What is the role of epidural analgesia in abdominal surgery? Cont Educ Anaesth Crit Care Pain. 2014;14(5):2249. doi:10.1093/bjaceaccp/mkt062.

12. Holte $\mathrm{K}$, Kehlet $\mathrm{H}$. Effect of postoperative epidural analgesia on surgical outcome. Minerva Anestesiol. 2002;68(4):157-61. [PubMed: 12024074].

13. Goldman LEE. Cardiac risks and complications of noncardiac surgery. Ann Surg. 1983;198(6):780-91. doi: 10.1097/00000658-19831200000019. [PubMed Central: PMC1353230].

14. Liu S, Carpenter RL, Neal JM. Epidural anesthesia and analgesia. Their role in postoperative outcome. Anesthesiology. 1995;82(6):1474-506. doi: 10.1097/00000542-199506000-00019. [PubMed: 7793661].

15. Sydow FW. The influence of anesthesia and postoperative analgesic management of lung function. Acta Chir Scand Suppl.1989;550:159-65. discussion 165-8. [PubMed: 2652967].

16. Fotiadis RJ, Badvie S, Weston MD, Allen-Mersh TG. Epidural analgesia in gastrointestinal surgery. Br J Surg. 2004;91(7):828-41. doi: 10.1002/bjs.4607. [PubMed: 15227688].

17. Kehlet H. The stress response to surgery: Release mechanisms and the modifying effect of pain relief. Acta Chir Scand Suppl. 1989;550:22-8. [PubMed: 2652970].

18. Moraca RJ, Sheldon DG, Thirlby RC. The role of epidural anesthesia and analgesia in surgical practice. Ann Surg. 2003;238(5):663-73. doi: 10.1097/01.sla.0000094300.36689.ad. [PubMed: 14578727]. [PubMed Central: PMC1356143].

19. Holte $K$, Kehlet H. Epidural analgesia and risk of anastomotic leakage. Reg Anesth Pain Med. 2001;26(2):111-7. doi: 10.1053/rapm.2001.21241. [PubMed: 11251133].

20. Borromeo CJ, Stix MS, Lally A, Pomfret EA. Epidural catheter and increased prothrombin time after right lobe hepatectomy for living donor transplantation. Anesth Analg. 2000;91(5):1139-41. doi: 10.1097/00000539-200011000-00018. [PubMed: 11049898].

21. Pelton JJ, Hoffman JP, Eisenberg BL. Comparison of liver function tests after hepatic lobectomy and hepatic wedge resection. Am Surg. 1998;64(5):408-14. [PubMed: 9585773].

22. Cunningham JD, Fong Y, Shriver C, Melendez J, Marx WL, Blumgart LH. One hundred consecutive hepatic resections. Blood loss, transfusion, and operative technique. Arch Surg. 1994;129(10):1050-6. doi: 10.1001/archsurg.1994.01420340064011. [PubMed: 7944934].

23. Weinberg L, Gilbee E, Tan C, Scurrah N, Chen G, McNicol L, et al. Analgesia practice for pancreaticoduodenectomy (Whipple's procedure): Is it time to reconsider practice? Austin Hospital, Melbourne: Departments of Anaesthesia \& Surgery; 2013.

24. Meylan N, Elia N, Lysakowski C, Tramer MR. Benefit and risk of intrathecal morphine without local anaesthetic in patients undergoing major surgery: Meta-analysis of randomized trials. Br J Anaesth. 2009;102(2):156-67. doi: 10.1093/bja/aen368. [PubMed: 19151046].

25. Law CJ, Visser EJ. Unconsciousness and severe respiratory depression following intrathecal morphine analgesia for lumbar spinal surgery. Acute Pain. 2007;9(3):163-7. doi: 10.1016/j.acpain.2007.05.001.

26. Sakowska M, Docherty E, Linscott D, Connor S. A change in practice from epidural to intrathecal morphine analgesia for hepatopancreato-biliary surgery. World J Surg. 2009;33(9):1802-8. doi: 10.1007/s00268-009-0131-2. [PubMed: 19548026].

27. Moher D, Liberati A, Tetzlaff J, Altman DG, Prisma Group. Preferred reporting items for systematic reviews and meta-analyses: The PRISMA statement. PLOS Med. 2009;6(7). e1000097. doi: 10.1371/journal.pmed.1000097. [PubMed: 19621072]. [PubMed Central: PMC2707599]. 
28. Downs SH, Black N. The feasibility of creating a checklist for the assessment of the methodological quality both of randomised and non-randomised studies of health care interventions. $J$ Epidemiol Community Health. 1998;52(6):377-84. doi: 10.1136/jech.52.6.377. [PubMed: 9764259]. [PubMed Central: PMC1756728].

29. Devys JM, Mora A, Plaud B, Jayr C, Laplanche A, Raynard B, et al. Intrathecal + PCA morphine improves analgesia during the first $24 \mathrm{hr}$ after major abdominal surgery compared to PCA alone. Can J Anaesth. 2003;50(4):355-61. doi: 10.1007/BF03021032. [PubMed: 12670812].

30. De Pietri L, Siniscalchi A, Reggiani A, Masetti M, Begliomini B, Gazzi $\mathrm{M}$, et al. The use of intrathecal morphine for postoperative pain relief after liver resection: A comparison with epidural analgesia. Anesth Analg. 2006;102(4):1157-63. doi: 10.1213/01.ane.0000198567.85040.ce. [PubMed: 16551916].

31. Roy JD, Massicotte L, Sassine MP, Seal RF, Roy A. A comparison of intrathecal morphine/fentanyl and patient-controlled analgesia with patient-controlled analgesia alone for analgesia after liver resection. Anesth Analg. 2006;103(4):990-4. doi: 10.1213/01.ane.0000238040.41872.7e. [PubMed: 17000818].

32. Duncan MA, Savage J, Tucker AP. Prospective audit comparing intrathecal analgesia (incorporating midazolam) with epidural and intravenous analgesia after major open abdominal surgery. Anaesth Intensive Care. 2007;35(4):558-62. doi: 10.1177/0310057X0703500415. [PubMed: 18020075].

33. Ko JS, Choi SJ, Gwak MS, Kim GS, Ahn HJ, Kim JA, et al. Intrathecal morphine combined with intravenous patient-controlled analgesia is an effective and safe method for immediate postoperative pain control in live liver donors. Liver Transpl. 2009;15(4):381-9. doi: 10.1002/It.21625. [PubMed: 19326422].

34. Koea JB, Young Y, Gunn K. Fast track liver resection: The effect of a comprehensive care package and analgesia with single dose intrathecal morphine with gabapentin or continuous epidural analgesia. HPB Surg. 2009;2009:271986. doi: 10.1155/2009/271986. [PubMed: 20029637]. [PubMed Central: PMC2796218].

35. Lee SH, Gwak MS, Choi SJ, Park HG, Kim GS, Kim MH, et al. Prospective, randomized study of ropivacaine wound infusion versus intrathecal morphine with intravenous fentanyl for analgesia in living donors for liver transplantation. Liver Transpl. 2013;19(9):1036-45. doi: 10.1002/lt.23691. [PubMed: 23788468].

36. Kasivisvanathan R, Abbassi-Ghadi N, Prout J, Clevenger B, Fusai GK, Mallett SV. A prospective cohort study of intrathecal versus epidural analgesia for patients undergoing hepatic resection. HPB (Oxford). 2014;16(8):768-75. doi: 10.1111/hpb.12222. [PubMed: 24467320].
[PubMed Central: PMC4113260].

37. Dichtwald S, Ben-Haim M, Papismedov L, Hazan S, Cattan A, Matot I. Intrathecal morphine versus intravenous opioid administration to impact postoperative analgesia in hepato-pancreatic surgery: A randomized controlled trial.JAnesth. 2017;31(2):237-45. doi 10.1007/s00540-016-2286-y. [PubMed: 27885425].

38. Revie EJ, Massie LJ, McNally SJ, McKeown DW, Garden OJ, Wigmore SJ. Effectiveness of epidural analgesia following open liver resection. HPB (Oxford). 2011;13(3):206-11. doi: 10.1111/j.1477-2574.2010.00274.x. [PubMed: 21309939]. [PubMed Central: PMC3048973].

39. Jones RM, Moulton CE, Hardy KJ. Central venous pressure and its effect on blood loss during liver resection. BrJ Surg. 1998;85(8):1058-60. doi: 10.1046/j.1365-2168.1998.00795.x. [PubMed: 9717995].

40. Walsh SR, Walsh CJ. Intravenous fluid-associated morbidity in postoperative patients. Ann $R$ Coll Surg Engl. 2005;87(2):126-30. doi 10.1308/147870805X28127. [PubMed: 15826425]. [PubMed Central: PMC1963879].

41. Aloia TA, Kim BJ, Segraves-Chun YS, Cata JP, Truty MJ, Shi Q, et al. A randomized controlled trial of postoperative thoracic epidural analgesia versus intravenous patient-controlled analgesia after major hepatopancreatobiliary surgery. Ann Surg. 2017;266(3):545-54. doi 10.1097/SLA.0000000000002386. [PubMed: 28746153]. [PubMed Central: PMC5784834]

42. Moen V, Dahlgren N, Irestedt L. Severe neurological complications after central neuraxial blockades in Sweden 1990-1999. Anesthesiology. 2004;101(4):950-9. doi: 10.1097/00000542-200410000-00021. [PubMed: 15448529].

43. Pan PH, Bogard TD, Owen MD. Incidence and characteristics of failures in obstetric neuraxial analgesia and anesthesia: A retrospective analysis of 19,259 deliveries. Int J Obstet Anesth. 2004;13(4):227-33. doi: 10.1016/j.ijoa.2004.04.008. [PubMed: 15477051].

44. Pratt WB, Steinbrook RA, Maithel SK, Vanounou T, Callery MP, Vollmer CM Jr. Epidural analgesia for pancreatoduodenectomy: A critical appraisal.J Gastrointest Surg. 2008;12(7):1207-20.doi:10.1007/s11605-0080467-1. [PubMed: 18264686].

45. Gehling M, Tryba M. Risks and side-effects of intrathecal morphine combined with spinal anaesthesia: A meta-analysis. Anaesthesia. 2009;64(6):643-51. doi: 10.1111/j.1365-2044.2008.05817.x. [PubMed: 19462494]

46. Bailey PL, Rhondeau S, Schafer PG, Lu JK, Timmins BS, Foster W, et al. Dose-response pharmacology of intrathecal morphine in human volunteers. Anesthesiology. 1993;79(1):49-59. discussion 25A. doi 10.1097/00000542-199307000-00010. [PubMed: 8342828]. 